Rectally shed SARS-CoV-2 lacks infectivity: time to rethink faecal-oral transmission?

\section{Rune M. Pedersen, Ditte S. Tornby, Line L. Bang, Lone W. Madsen (1), Marianne N. Skov (D), Thøger G. Jensen (D), Isik S. Johansen (D) and}

Thomas E. Andersen (1)

We read with interest the Perspective by Guo et al. (Guo, M. et al. Potential intestinal infection and faecal-oral transmission of SARS-CoV-2. Nat. Rev. Gastroenterol. Hepatol. 18, 269-283(2021) $)^{1}$, on the potential faecaloral transmission of severe acute respiratory syndrome coronavirus 2 (SARS-CoV-2). A comprehensive list of reasons to substantiate that such a transmission route is probable was presented, including the well-documented shedding of viral RNA in faeces, epidemiological reports, and laboratory and animal tests. Viral debris and RNA are often found in large quantities in faeces from patients with COVID-19 as most of the viral material produced in the airways is cleared through swallowing. Furthermore, as argued by Guo et al. ${ }^{1}$, studies indicate that intestinal infection by SARS-CoV-2 might contribute to the viral material shed in faeces.

In either case, it is important to emphasize that for the rectally shed virus to transmit to other humans it must retain its infectivity when it leaves the body. Proof in support of this property is obtained by successful isolation of the virus; that is, successful propagation of the sampled virus in laboratory cell culture. Guo et al. ${ }^{1}$ refer to four studies in support of infectious virus particles being successfully isolated from patient's faeces ${ }^{2-5}$. However, three of these studies base their conclusion of successful propagation of SARS-CoV-2 on microscopy observations alone ${ }^{2-4}$, which is inadequate as it requires additional substantiation by quantitative analysis (such as reverse transcription PCR, RT-PCR) to conclude that virions have increased in number upon culturing ${ }^{6,7}$. All three papers use electron microscopy to show what appears to be intact SARS-CoV-2 particles in culture supernatants ${ }^{2-4}$, but identifying SARS-CoV-2 in complex specimens in this way is difficult and error prone ${ }^{8,9}$, and, even if visually intact virions are identified, it does not provide evidence for infectious capacity. Notably, only Zhou et al. confirmed viral propagation by RT-PCR ${ }^{5}$; a result that was obtained for a single faecal sample ${ }^{5}$ and is, to the best of our knowledge, the only valid evidence reported in the literature that supports retained infectivity of rectally shed SARS-CoV-2.

One additional study has attempted culturing of rectally shed SARS-CoV-2 with the use of confirmatory RT-PCR. The comprehensive study by Wölfel et al. analysed 13 faecal samples from four patients during their course of COVID-19 and concluded that none of the samples contained culturable virus ${ }^{10}$. Results from our hospital are in line with this outcome as we have, to date, not been able to culture rectally shed SARS-CoV-2 from patients with COVID-19 of varying disease course (R.M.P., D.S.T., L.L.B., L.W.M., M.N.S., T.G.J., I.S.J. and T.E.A., unpublished data).

The potential existence of a faecal-oral transmission route has gained widespread attention. We would like to add to this discussion that the evidence in support of the key property that allows such a transmission, that rectally shed SARS-CoV-2 is infectious, remains weak and in our view inconclusive. New data to support this capacity have not emerged since the early reports on this topic, overall questioning whether such transmission is plausible and plays a substantial part in the dissemination of the virus.

There is a reply to this letter by Guo, M. et al. Reply to: Rectally shed SARS-CoV-2 lacks infectivity: time to rethink faecal-oral transmission? Nat. Rev. Gastroenterol. Hepatol. https://doi.org/10.1038/s41575-021-00503-8 (2021).

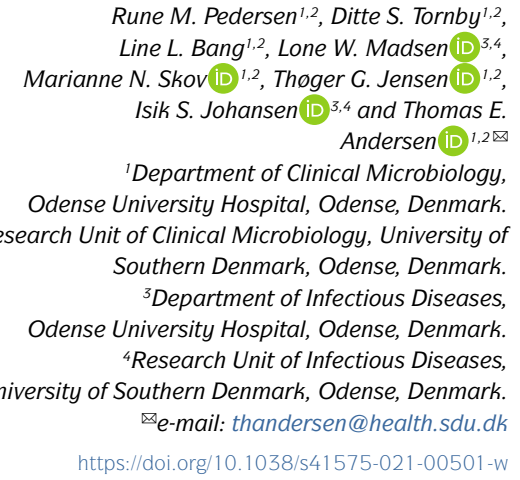

1. Guo, M., Tao, W., Flavell, R. A. \& Zhu, S. Potential intestinal infection and faecal-oral transmission of SARS-CoV-2. Nat. Rev. Gastroenterol. Hepatol. 18 269-283 (2021).

2. Zhang, Y. et al. Isolation of 2019-nCoV from a stool specimen of a laboratory-confirmed case of the coronavirus disease 2019 (COVID-19). China CDC Wkly 2, 123-124 (2020).

3. Xiao, F. et al. Infectious SARS-CoV-2 in feces of patient with severe COVID-19. Emerg. Infect. Dis. 26, 1920-1922 (2020).

4. Wang, W. et al. Detection of SARS-CoV-2 in different types of clinical specimens. JAMA 323, 1843-1844 (2020).

5. Zhou, J. et al. Infection of bat and human intestinal organoids by SARS-CoV-2. Nat. Med. 26, 1077-1083 (2020).

6. Folgueira, M. D. et al. Prolonged SARS-CoV-2 cell culture replication in respiratory samples from patients with severe COVID-19. Clin. Microbiol. Infect. 27, 886-891 (2021)

7. Arons, M. M. et al. Presymptomatic SARS-CoV-2 infections and transmission in a skilled nursing facility. N. Engl. J. Med. 382, 2081-2090 (2020).

8. Goldsmith, C. S. et al. Electron microscopy of SARS-CoV-2: a challenging task. Lancet 395, e99 (2020).

9. Miller, S. E. \& Goldsmith, C. S. Caution in identifying coronaviruses by electron microscopy. J. Am. Soc. Nephrol. 31, 2223-2224 (2020).

10. Wölfel, R. et al. Virological assessment of hospitalized patients with COVID-2019. Nature 581, 465-469 (2020).

Competing interests

The authors declare no competing interests.

\title{
Reply to: Rectally shed SARS-CoV-2 lacks infectivity: time to rethink faecal-oral transmission?
}

\section{Meng Guo (D), Wanyin Tao, Richard A. Flavell(D) and Shu Zhu(D)}

To determine whether severe acute respiratory syndrome coronavirus 2 (SARS-CoV-2) can establish a faecal-oral transmission route, it is essential to confirm that infectious virus particles are shed in faeces from patients with COVID-19. We would like to thank Pedersen et al. for their Correspondence on our Perspective (Guo, M. et al. Potential intestinal infection and faecal-oral transmission of SARS-CoV-2. Nat. Rev. Gastroenterol. Hepatol. 18, 269-283 (2021)) $)^{1}$, which raises some important issues (Pedersen et al. Rectally shed SARS-CoV-2 lacks infectivity: time to rethink faecal-oral transmission? Nat. Rev. Gastroenterol. Hepatol. https://doi. org/10.1038/s41575-021-00501-W (2021))2. 
Our Perspective refers to four studies that discuss isolation of infectious virus particles from patient faeces ${ }^{3-6}$. Two of these studies ${ }^{5,6}$ only reported data obtained by electron microscopy, which are indeed insufficient to determine infectivity, as argued by Pedersen et al. ${ }^{2}$. However, Zhou and colleagues reported the extraction of infectious virus particles from a patient with COVID-19 based on increases in viral load in human intestinal organoids ${ }^{4}$. Also, Xiao et al. reported isolation of SARS-CoV-2 particles from two of three stool samples ${ }^{3}$; the viral isolates, first observed by electron microscopy, could successfully infect Vero cells (confirmed by immunofluorescence staining $)^{3}$. More recently, when three viral isolates were extracted from stool samples of patients with COVID-19 and added to Vero cells ${ }^{7}$, the culture supernatants tested positive for SARS-CoV-2 by quantitative reverse transcription PCR. The infectious viral particles were collected from culture supernatant viral stock, then frozen and subsequently thawed stock were able to infect Vero and Calu-3 (human lung adenocarcinoma) cells. Two of the three viral faecal isolates were more infectious in cells than viral isolates extracted from nasopharyngeal swabs and sputum in vitro ${ }^{7}$. These results strongly suggested that infectious virus could be shed in faecal samples from patients with COVID-19.
Notably, three of five studies mentioned here also included patient information ${ }^{3,6,7}$, and all of the viral isolates in these studies were extracted from patients with severe disease. Without more information, we cannot exclude the possibility that one study mentioned by Pederson et al. that failed to isolate infectious SARS-CoV-2 from stool could be attributable to a mild disease course in those patients ${ }^{8}$. Moreover, Xiao et al. failed to isolate virus from stool at later time points, indicating the absence of infectious virus, with only viral fragments apparently shed in faeces during late stages of the disease ${ }^{3}$. Thus, disease severity and time course for sampling could be determining factors in the isolation of infectious viral particles.

Research in animals might also provide valuable insights. One study investigating SARS-CoV-2 infection in rhesus macaques reported the successful isolation of infectious viruses from faeces, and confirmed this finding by electron microscopy and TCID50 (median tissue culture infective dose) assays $\left(10^{5} / \mathrm{ml}\right)^{9}$. Similarly, a study of SARS-CoV-2 infection in ferrets showed that oral inoculation with faecal supernatants of infected specimens could establish infection in naive ferrets ${ }^{10}$. These animal studies therefore demonstrated the presence of infectious virus in the faeces of COVID-19 animal models. Although research has suggested

Table 1 | Research on the infectivity of virus shedding in faecal samples in human and animal studies

\begin{tabular}{|c|c|c|c|c|c|c|}
\hline Reference & $\begin{array}{l}\text { Electron } \\
\text { microscopy }\end{array}$ & RT-PCR & $\begin{array}{l}\text { Cytopathic } \\
\text { effects }^{a}\end{array}$ & $\begin{array}{l}\text { Immuno- } \\
\text { fluorescence }^{\text {b }}\end{array}$ & TCID50 & $\begin{array}{l}\text { Faecal } \\
\text { supernatants } \\
\text { inoculating } \\
\text { naive animals }\end{array}$ \\
\hline \multicolumn{7}{|c|}{ Clinical studies } \\
\hline Yao et al. ${ }^{7}$ & - & + & + & + & + & - \\
\hline Xiao et al. ${ }^{3}$ & + & - & + & + & - & - \\
\hline Zhou et al. ${ }^{4}$ & - & + & - & - & - & - \\
\hline Wang et al. ${ }^{5}$ & + & - & - & - & - & - \\
\hline Zhang et al. $^{6}$ & + & - & - & - & - & - \\
\hline Wölfel et al. ${ }^{8}$ & \multicolumn{6}{|c|}{ Failed to isolate in mild cases } \\
\hline \multicolumn{7}{|c|}{ Animal studies } \\
\hline Jiao et al. ${ }^{9}$ & - & - & - & - & + & - \\
\hline Kim et al. ${ }^{10}$ & - & - & - & - & - & + \\
\hline
\end{tabular}

that infectious viral particles can be isolated from both humans and animals, based on the available evidence (TABLE 1), a clear faecal-oral transmission route has not been established for SARS-CoV-2.

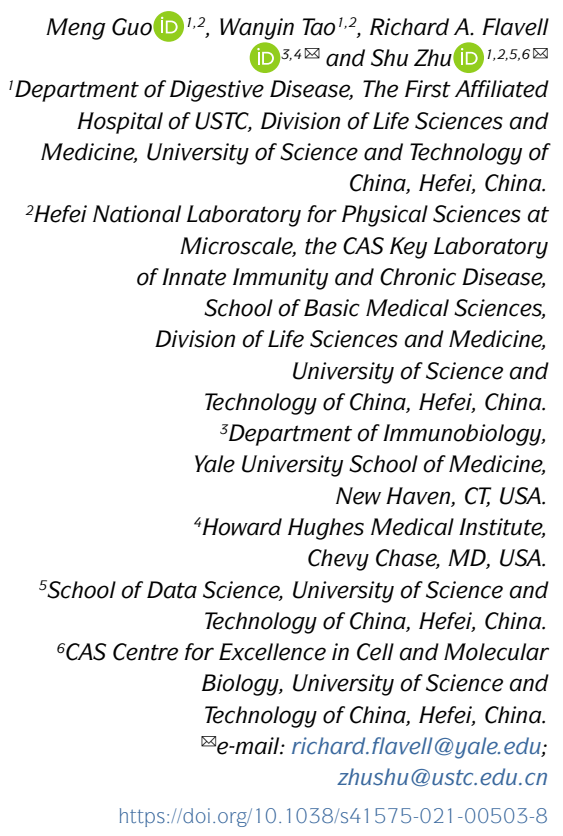

1. Guo, M., Tao, W., Flavell, R. A. \& Zhu, S. Potential intestinal infection and faecal-oral transmission of SARS-CoV-2. Nat. Rev. Gastroenterol. Hepatol. 18 269-283 (2021)

2. Pedersen, R. M. et al. Rectally shed SARS-CoV-2 lacks infectivity: time to rethink faecal-oral transmission? Nat. Rev. Gastroenterol. Hepatol. https://doi.org/10.1038/s41575-021-00501-w (2021).

3. Xiao, F. et al. Infectious SARS-CoV-2 in feces of patient with severe COVID-19. Emerg. Infect. Dis. 26, 1920-1922 (2020).

4. Zhou, J. et al. Infection of bat and human intestinal organoids by SARS-CoV-2. Nat. Med. 26, 1077-1083 (2020).

5. Wang, W. et al. Detection of SARS-CoV-2 in different types of clinical specimens. JAMA 323, 1843-1844 (2020).

6. Zhang, Y. et al. Isolation of 2019-nCoV from a Stool specimen of a laboratory confirmed case of the coronavirus disease 2019 (COVID-19). China CDC Wkly. 2, 123-124 (2020).

7. Yao, H. et al. Patient-derived SARS-CoV-2 mutations impact viral replication dynamics and infectivity in vitro and with clinical implications in vivo. Cell Discov. 6, 76 (2020).

8. Wölfel, R. et al. Virological assessment of hospitalized patients with COVID-2019. Nature 581, 465-469 (2020).

9. Jiao, L. et al. The gastrointestinal tract is an alternative route for SARS-CoV-2 infection in a nonhuman primate model. Gastroenterology 160 , 1647-1661 (2021).

10. Kim, Y. I. et al. Infection and rapid transmission of SARS-CoV-2 in ferrets. Cell Host Microbe 27, 704-709 (2020).

\section{Competing interests}

The authors declare no competing interests. 